\title{
A Griffith Crack at the Interface of an Isotropic and Orthotropic Half Space Bonded Together
}

\author{
A. K. Awasthi", Rachna, Harpreet Kaur
}

Department of Mathematics, Lovely Professional University, India

Received April 25, 2021; Revised December 9, 2021; Accepted December 27, 2021

\section{Cite This Paper in the following Citation Styles}

(a): [1] A. K. Awasthi, Rachna, Harpreet Kaur, "A Griffith Crack at the Interface of an Isotropic and Orthotropic Half Space Bonded Together," Mathematics and Statistics, Vol. 10, No. 1, pp. 166 - 175, 2022. DOI: 10.13189/ms.2022.100115.

(b): A. K. Awasthi, Rachna, Harpreet Kaur (2022). A Griffith Crack at the Interface of an Isotropic and Orthotropic Half Space Bonded Together. Mathematics and Statistics, 10(1), 166 - 175. DOI: 10.13189/ms.2022.100115.

Copyright $\odot 2022$ by authors, all rights reserved. Authors agree that this article remains permanently open access under the terms of the Creative Commons Attribution License 4.0 International License

\begin{abstract}
In the past 53 years, many efforts have been contributed to develop and demonstrate the properties of reinforced composite materials. The ever-increasing use of composite materials through engineering structures needs the proper analysis of the mechanical response of these structures. In the proposed work, we have an exact form of Stress components and Displacement components to a Griffith crack at the interface of an Isotropic and Orthotropic half-space bounded together. The expression was evaluated in the vicinity of crack tips by using Fourier transform method but here these components have been evaluated with the help of Fredholm integral equations and then reduce to the coupled Fredholm integral equations. In this paper, we use the problem of Lowengrub and Sneddon and reduce it to dual integral equations. Solution of these equations through the use of the method of Srivastava and Lowengrub is reduced to coupled Fredholm integral equation. Further reduces the problem to decoupled Fredholm integral equation of 2 nd kind. We get the solution of dual integral equations and the problem is reduced to coupled Fredholm integral equation. We find the solution of the Fredholm integral equation and reduce it to decoupled Fredholm integral equation of 2 nd kind. The Physical interest in fracture design criterion is due to Stress and crack opening Displacement components. In the end, we can easily calculate the Stress components and Displacement components in the exact form.
\end{abstract}

Keywords Stress Components, Displacement Components, Fourier Transform, Fredholm Integral Equation, Coupled Fredholm Integral Equation

\section{Introduction}

The exact expressions of the displacement and the stress components in the vicinity of crack-tips at the common interface of the one isotropic and other one orthotropic half-plane are evaluated by using Fourier transform method. A square root singularity is observed in these components.

Williams [1,3] solved the problem of the stress and displacement fields in the neighbourhood of crack tips by using the complex variable method. Erdogan [4] extended the application of the above method to more Griffith-cracks. Rice and Sih [5] used R-integral approach to solve the problem of cracks in the bounded dissimilar materials. Willis [18] in his research endeavor develop the fractured design parameters for interfacial cracks. Dwivedi et al. $[13,1,16]$ solved the problems of dissimilar media containing cracks.

Hydro-static pressure over the structure is transformed to constant pressure at crack faces. Lowengrub and Sneddon [11] solved the problem of a Griffith crack at the interface of two bounded isotropic homogeneous half-planes by using transform techniques. The Hilbert problem is reduced by Muskhelishvili [6]. The solutions involve logarithmic violent oscillations and the square root singularity. Borai and Nadi [21] discussed some singular and non-linear singular equations in Banach space and Fourier transform used for Cauchy problems for parabolic and hyperbolic partial differential equations. Leontiev [22] described surface value problem with some boundary problems by linear and finite Fourier series.

In the proposed work the problem is formulated on the 
lines of [11]. However, not reduce Hilbert problem. The problem is reduced to dual integral equations whose solutions through the use of methods of Srivastava and Lowengrub [8] are reduced to coupled Fredholm integral equations.

The further simplification reduces the problem to decoupled Fredholm integral equations of the second kind.
The problem of proposed work on a mixed boundary problem of the following type;

An interior Griffith crack occupies the region $y=0,0 \leq|x| \leq b$ and boundary conditions, see Fig. 1. and

Fig. 2 .

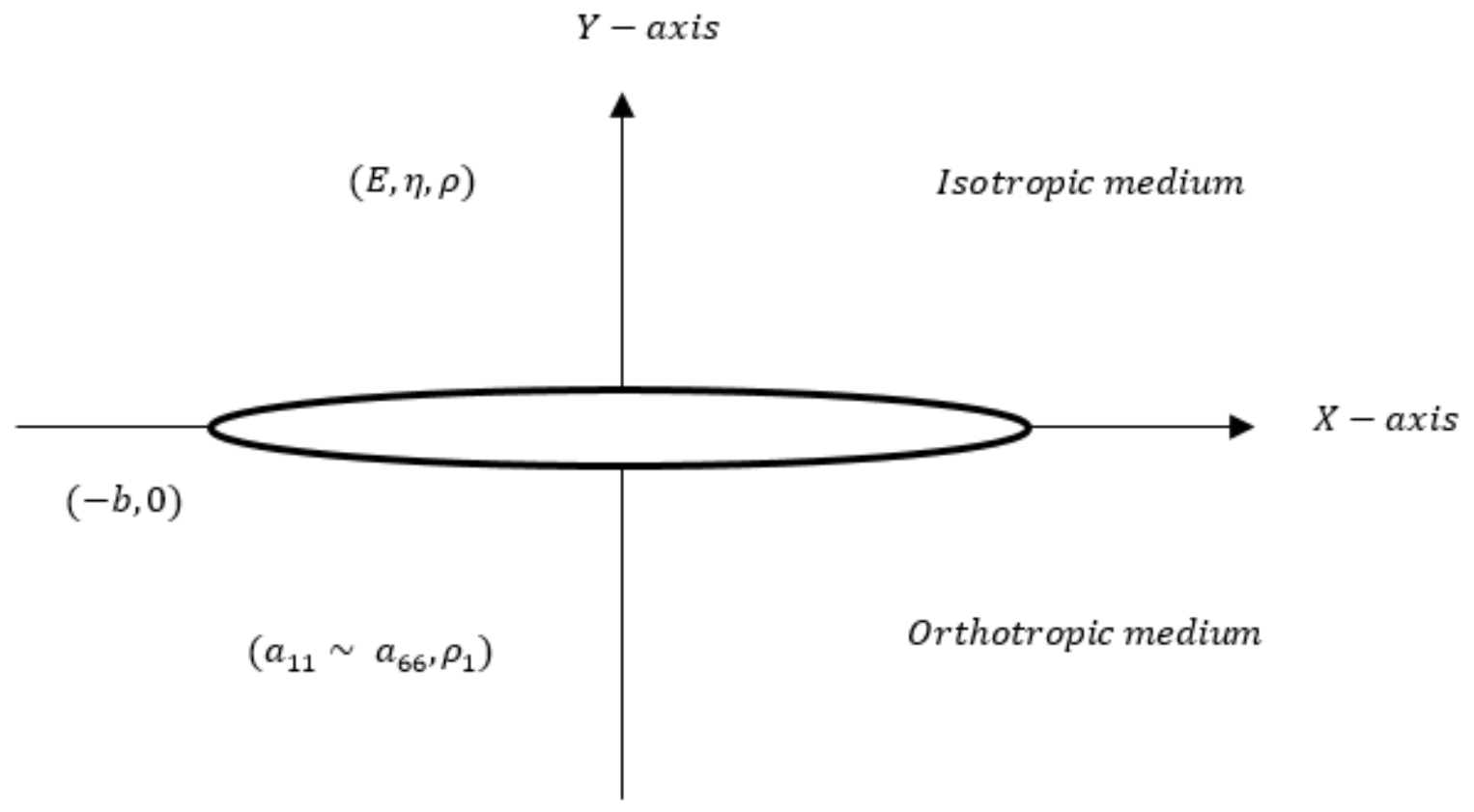

Figure 1. Geometry of the problem with a Griffith Crack

$$
Y-\text { axis }
$$

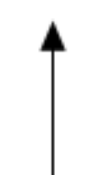

Isotropic medium

$$
p_{1}(x)
$$

$$
\left(a_{11} \sim a_{66}, \rho_{1}\right)
$$$$
p_{2}(x)
$$$$
\text { Orthotropic medium }
$$

Figure 2. Geometry with boundary conditions 
The problem of proposed work is a mixed boundary problem of an interior Griffith Crack occupies on the region $y=0,0 \leq|x| \leq b$ shown in figure 1 and 2 with boundary conditions and continuity conditions.

Boundary condition,

$$
\sigma_{x y}\left(x, 0^{+}\right)=\sigma_{x y}\left(x, 0^{-}\right)=0,0 \leq|x| \leq b
$$

Suppose, $\sigma_{x y}\left(x, 0^{+}\right)=-p_{1}(x)$ and $\sigma_{x y}\left(x, 0^{-}\right)=-p_{2}(x)$

Continuity conditions,

$$
\begin{gathered}
\sigma_{x y}\left(x, 0^{+}\right)=\sigma_{x y}\left(x, 0^{-}\right), b \leq|x|<\infty \\
\sigma_{x y}\left(x, 0^{+}\right)=\sigma_{x y}\left(x, 0^{-}\right), b \leq|x|<\infty \\
u_{y}\left(x, 0^{+}\right)=u_{y}\left(x, 0^{-}\right), b \leq|x|<\infty \\
u_{x}\left(x, 0^{+}\right)=u_{x}\left(x, 0^{-}\right), b \leq|x|<\infty
\end{gathered}
$$

Where $u_{x}, u_{y}$ and $\sigma_{x x}, \sigma_{x y}, \sigma_{y y}$ are the components of displacement vector and component of stress vector, and physical quantity $\left(0^{+}\right)$and $\left(0^{-}\right)$for region $y>0$ and $y<0$.

Airy applied the stress function method for $y>0$ and Kushwaha applied stress function method for $y<0$, for solution at any general point $(x, y)$. In proposed work, property of symmetry applied on geometry of crack and medium.

The common interface passes through mid of the Griffith crack and perpendicular to $\mathrm{x}$ - axis. The isotropic homogeneous half-plane occupies the region $y>0$ and orthotropic homogeneous half-plane occupies the region $y<0$. Isotropic and orthotropic both planes are between the plain strain conditions and any type of physical quantities vanish, if

$$
\sqrt{x^{2}+y^{2}} \Rightarrow \infty
$$

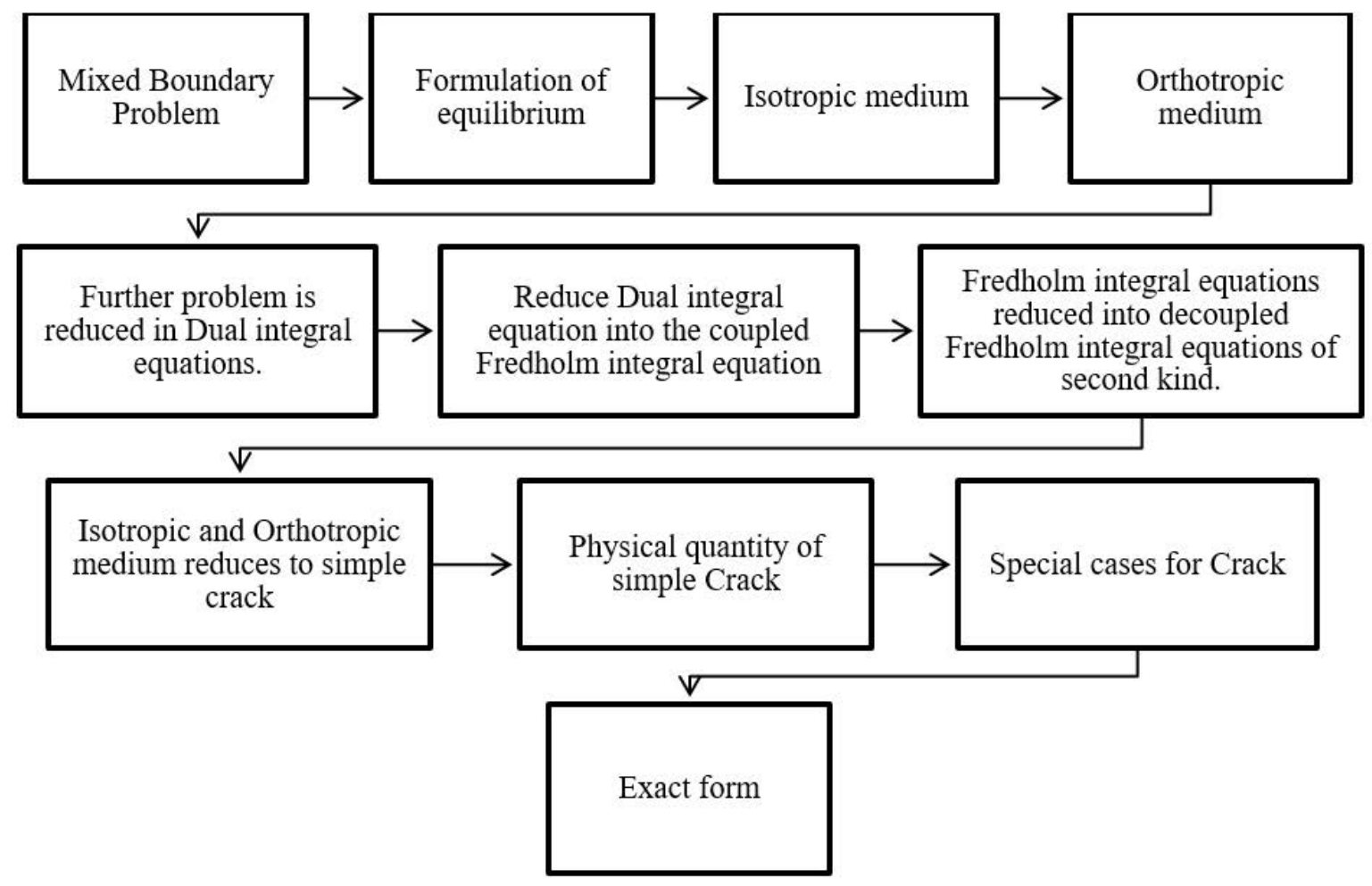

Flow chart of Process 


\section{Formulation}

The solution of equations of equilibrium,

$$
\begin{aligned}
& \frac{\partial \sigma_{x x}}{\partial x}+\frac{\partial \sigma_{x y}}{\partial y}=0 \\
& \frac{\partial \sigma_{x y}}{\partial x}+\frac{\partial \sigma_{x y}}{\partial y}=0
\end{aligned}
$$

$u_{y}(x, y)=2 \frac{(1+\eta)}{\pi E} \int_{0}^{\infty} \frac{\cos (\xi x)}{\xi^{2}}\left[\frac{(1-\eta) \partial^{3}}{\partial y^{3}} H(\xi, y)+\right.$

$\left.(\eta-2) \xi^{2} \frac{\partial}{\partial y} H(\xi, y)\right] d \xi$

In case of isotropic, $y>0$ for the absence of body force. i.e.

$$
u_{y}(x, y)=2 \frac{(1+\eta)}{\pi E} \int_{0}^{\infty} \frac{\sin (\xi x)}{\xi}\left[(1-\eta) \frac{\partial^{2} H}{\partial y^{2}}+\eta \xi^{2} H(\xi y)\right] d \xi
$$

Use $H(\xi, y)=[A(\xi)+y B(\xi)] e^{(-\xi y)}$, where A and B are arbitrary constants.

In case of orthotropic, $y<0$ for the absence of body force. i.e.

$$
\begin{aligned}
& u_{y}(x, y)=\int_{0}^{\infty} \frac{\cos (\xi x)}{\xi^{2}}\left[a_{11} G(\xi, y)-\xi^{2}\left(a_{12}+a_{66}\right) G(\xi, y)\right] d \xi \\
& u_{x}(x, y)=\int_{0}^{\infty} \frac{\sin (\xi x)}{\xi}\left[a_{11} G(\xi, y)-a_{12} \xi^{2} G(\xi, y)\right] d \xi
\end{aligned}
$$

$\operatorname{Used} G(\xi, y)=\frac{1}{\left(r_{1}-r_{2}\right)}\left[\left\{\left(r_{1}-r_{2}\right) C(\xi)-D(\xi)\right\} e^{r_{1} \xi y}+D(\xi) e^{r_{2} \xi y}\right]$.

Where C, D are two arbitrary constants and $\eta$ and $\eta_{2}$ are two roots of $r^{4}+B_{1} r^{2}+B^{2}=0$ Where $B_{1}=\frac{2\left(a_{12}+a_{66}\right)}{a_{11}}$ and $B_{2}=\frac{a_{22}}{a_{11}}$ and $a_{11}, a_{12}, a_{66}$ are elastic constants of orthotropic medium. The stress strain relations are:

(a) Isotropic Case:-

$$
\begin{gathered}
2 \mu e_{x x}=(1-\eta) \sigma_{x x}-\eta \sigma_{y y} \\
2 \mu e_{y y}=(1-\eta) \sigma_{y y}-\eta \sigma_{x x} \\
2 \mu e_{x y}=\sigma_{x y}
\end{gathered}
$$

Where $\mu$ and $\eta$ are modulus of rigidity and poisson's ratio of isotropic medium and $\left(e_{x x}, e_{x y}, e_{y y}\right)$ are elastic strains.

(b) Orthotropic Case:-

$$
\begin{aligned}
& \frac{\partial u_{x}}{\partial x}=a_{11} \sigma_{x x}+a_{12} \sigma_{y y} \\
& \frac{\partial u_{y}}{\partial y}=a_{12} \sigma_{x x}+a_{22} \sigma_{y y} \\
& \frac{\partial u_{x}}{\partial y}+\frac{\partial u_{y}}{\partial x}=a_{66} \sigma_{x y}
\end{aligned}
$$

Fourier inversions of the functions applied on equation of equilibrium, eq. (6), (7) and continuity conditions eq. (3), (4). Then,

$$
\begin{gathered}
\xi r_{1} C(\xi)-D(\xi)+B(\xi)-\xi A(\xi)=0 \\
A(\xi)-C(\xi)=\frac{P_{1}(\xi)}{\xi^{2}}
\end{gathered}
$$

With $P_{1}(\xi)=\frac{2}{\pi} \int_{0}^{b}\left[p_{1}(x)-p_{2}(x)\right] \cos \xi x d x$, where A, B, C, D are four arbitrary constant. Use of a continuity conditions eq. (4), (5) to find the constants which are not eliminated. Thus get,

$\int_{0}^{\infty} \xi \sin (\xi x)\left[K_{5} C(\xi)+K_{6} D(\xi)\right] d \xi=P_{4}(x), B \leq x<\infty$ (12)

$\int_{0}^{\infty} \xi \cos (\xi x)\left[K_{7} C(\xi)+K_{8} D(\xi)\right] d \xi=P_{5}(x), b \leq x<\infty$

Where,

$$
\begin{gathered}
K_{0}=\frac{2(1+\eta)}{\pi E} \\
K_{1}=r_{1}^{2} a_{11}-a_{12} \\
K_{2}=r_{1} a_{11} \\
K_{3}=r_{1}\left(a_{11} r_{1}^{2}-a_{12}-a_{66}\right) \\
K_{4}=a_{11} r_{2}^{2}-a_{12}-a_{66} \\
K_{5}=K_{0}-K_{1}+2 K_{0}(1-\eta)\left(1-r_{1}\right) \\
K_{6}=K_{2}-2 K_{0}(1-\eta) \\
K_{7}=K_{0}\left\{1-2(1-\eta)\left(1-r_{1}\right)\right\}+K_{3} \\
K_{8}=2 K_{0}(1-\eta)+K_{4} \\
P_{4}(x)=\int_{0}^{\infty} \frac{\left.P_{1}(\xi) \sin (\xi x) d \xi\right)}{\xi} \\
P_{5}(x)=\int_{0}^{\infty} \frac{\left.P_{1}(\xi) \cos (\xi x) d \xi\right)}{\xi}
\end{gathered}
$$

Now to evaluate two more expressions are 


$$
\begin{gathered}
\sigma_{x y}\left(x, 0^{+}\right)+\sigma_{x y}\left(x, 0^{-}\right)=0,0 \leq x<b \\
\sigma_{y y}\left(x, 0^{+}\right)+\sigma_{y y}\left(x, 0^{-}\right)=\left(p_{1}(x)+p_{2}(x)\right), 0 \leq x<b
\end{gathered}
$$

These relation yield,

$$
\begin{gathered}
\int_{0}^{\infty} \xi^{2} \sin (\xi x) C(\xi) d \xi=\frac{1}{1+r_{1}} \\
\int_{0}^{\infty} \bar{P}_{1}(\xi) \sin (\xi x) d \xi=P_{6}(x), 0 \leq x<b \\
\int_{0}^{\infty} \xi^{2} \cos (\xi x) C(\xi) d \xi=P_{7}(x), 0 \leq x<b
\end{gathered}
$$

In equation (17) and (18), $P_{6}$ and $P_{7}$ are;

$$
\begin{gathered}
P_{6}(x)=\frac{1}{1+r_{1}} \int_{0}^{\infty} \bar{P}_{1}(\xi) \sin (\xi x) d \xi \\
P_{7}(x)=\frac{1}{2}\left(p_{1}(x)+p_{2}(x)\right)-\frac{1}{2} \int_{0}^{\infty} \bar{P}_{1}(\xi) \cos (\xi x) d \xi
\end{gathered}
$$

\subsection{Solution of Dual Integral Equations}

For solve the dual integral equations (12), (13) and (17), (18) assume, i.e.

$$
\begin{gathered}
\phi(\xi)=\xi\left[K_{5} C(\xi)+K_{6} D(\xi)\right] \\
\psi(\xi)=\xi\left[K_{7} C(\xi)+K_{8} D(\xi)\right]
\end{gathered}
$$

Where $\phi$ and $\psi$ are two arbitrary constants and used in dual integral equations (12), (13), (17), (18). We get

$$
\begin{gathered}
\int_{0}^{\infty} \phi(\xi) \sin (\xi x) d \xi=P_{4}(x), 0 \leq x<b \\
\int_{0}^{\infty} \psi(\xi) \cos (\xi x) d \xi=P_{5}(x), 0 \leq x<b \\
\int_{0}^{\infty} \xi\left[K_{10} \phi(\xi)-K_{11} \psi(\xi)\right] \cos (\xi x) d \xi=P_{7}(x), 0 \leq x<b \\
\int_{0}^{\infty} \xi\left[K_{12} \phi(\xi)-K_{13} \psi(\xi)\right] \sin (\xi x) d \xi=P_{6}(x), 0 \leq x<b
\end{gathered}
$$

Where,

$$
\begin{gathered}
K_{9}=K_{5}-K_{8}-K_{6} K_{7} \\
K_{10}=\frac{K_{8}}{K_{9}} \\
K_{11}=\frac{K_{7}}{K_{9}} \\
K_{12}=r_{1} K_{10}+K_{11} \\
K_{13}=r_{1} K_{11}-\frac{K_{5}}{K_{9}}
\end{gathered}
$$

Now we use the method of Srivastava and Lowengrub
[8]. Let us assume that

$$
\begin{gathered}
\xi \phi(\xi)=\frac{2}{\pi}\left[\int_{0}^{b} g(t) t-\cos \xi t d t-\int_{0}^{\infty} P_{4}(t) t-\cos \xi t d t\right] \\
\xi \psi(\xi)=\frac{2}{\pi}\left[\int_{0}^{b} h(t) \sin \xi t d t-\int_{0}^{\infty} P_{5}(t) t-\sin \xi t d t\right]
\end{gathered}
$$

Then equations (23), (24) are satisfied if

$$
\begin{aligned}
& \int_{0}^{b} g(t) d t=0 \\
& \int_{0}^{b} h(t) d t=0
\end{aligned}
$$

After substitution of these in equation (23), (24) then outcomes equations are;

$$
\begin{aligned}
& K_{10} g(x)+\frac{2 K_{11}}{\pi} \int_{0}^{b} \frac{t h(t) d t}{t^{2}-x^{2}}=-P_{8}(x), 0 \leq x<b \\
& \frac{2}{\pi} K_{12} \int_{0}^{b} \frac{x g(t) d t}{t^{2}-x^{2}}+K_{13} h(x)=P_{9}(x), 0 \leq x<b
\end{aligned}
$$

Where,

$$
\begin{aligned}
& P_{8}(x)=P_{7}(x)-\frac{4 K_{11}}{\pi} \int_{0}^{\infty} \frac{P_{4} d t}{t^{2}-x^{2}} \\
& P_{9}(x)=P_{6}(x)+\frac{4 K_{12}}{\pi} \int_{0}^{\infty} \frac{P_{5} d t}{t^{2}-x^{2}}
\end{aligned}
$$

Therefore, get some equations

$$
\begin{aligned}
& \int_{0}^{b} \frac{\operatorname{th}(t) d t}{t^{2}-x^{2}}=\frac{\pi}{2 K_{11}} P_{10}(x), 0 \leq x<b \\
& \int_{0}^{b} \frac{x g(t) d t}{t^{2}-x^{2}}=\frac{\pi}{2 K_{12}} P_{11}(x), 0 \leq x<b
\end{aligned}
$$

With

$$
\begin{aligned}
& P_{10}(x)=P_{8}(x)+K_{10} g(x) \\
& P_{11}(x)=P_{9}(x)-K_{13} h(x)
\end{aligned}
$$

Now, calculate the value of $h(t), g(t)$. i.e.

$$
\begin{aligned}
& h(t)=-\frac{1}{\pi K_{11}} \frac{1}{\sqrt{b^{2}-t^{2}}} \int_{0}^{b} \frac{P_{10}(x) \sqrt{b^{2}-x^{2}} d x}{x^{2}-t^{2}} \\
& g(t)=-\frac{1}{\pi K_{12}} \frac{1}{\sqrt{b^{2}-t^{2}}} \int_{0}^{b} x \frac{P_{11}(x) \sqrt{b^{2}-x^{2}} d x}{x^{2}-t^{2}}
\end{aligned}
$$

Thus, the problem is reduced to the solution of coupled Fredholm integral equations (29) and (30). In next section we shall obtain the solution of these equations.

\subsection{Solution of Fredholm Integral Equations}

Firstly, substitute the value of $P_{10}(x)$ and $P_{11}(x)$ in 
equation (29) and (30), we get

$$
\begin{aligned}
& h(t)=-P_{12}(t)-\frac{K_{10}}{\pi K_{11} \sqrt{b^{2}-t^{2}}} \int_{0}^{b} \frac{g(x) \sqrt{b^{2}-x^{2}} d x}{x^{2}-t^{2}} \\
& g(t)=-P_{13}(t)-\frac{K_{13}}{\pi K_{12} \sqrt{b^{2}-t^{2}}} \int_{0}^{b} \frac{h(x) \sqrt{b^{2}-x^{2}} d x}{x^{2}-t^{2}}
\end{aligned}
$$

In equation (31) and (32), use $P_{12}$ and $P_{13}$, i.e.

$$
\begin{aligned}
& P_{12}(t)=\frac{1}{\pi K_{11} \sqrt{b^{2}-t^{2}}} \int_{0}^{b} \frac{P_{8}(x) \sqrt{b^{2}-x^{2}} d x}{x^{2}-t^{2}} \\
& P_{13}(t)=\frac{1}{\pi K_{12} \sqrt{b^{2}-t^{2}}} \int_{0}^{b} \frac{x P_{9}(x) \sqrt{b^{2}-x^{2}} d x}{x^{2}-t^{2}}
\end{aligned}
$$

Now substituting for equation (32) into equation (31) and interchange the order of integrations and evaluating the integrals, we get

$$
h(t)=-P_{14}(t)+\frac{K_{10} K_{13}}{2 \pi^{2} K_{11} K_{12}} \int_{0}^{b} \frac{h(x) K_{0}(x, t) d x}{x^{2}-t^{2}}
$$

where $P_{14}(t)$ and $K_{0}(x, t)$ are,

$$
\begin{gathered}
P_{14}(t)=P_{12}(t)+\frac{t K_{10} K_{13}}{\pi^{2} K_{11} \sqrt{b^{2}-t^{2}}} \int_{0}^{b} \frac{P_{13}(x) \sqrt{b^{2}-x^{2}}}{x^{2}-t^{2}} d x \\
K_{0}(x, t)=\frac{1}{\sqrt{b^{2}-t^{2}}}\left[\log \left(\frac{b-t}{b+t}\right) \frac{b+x}{b-x}\right] \frac{\sqrt{b^{2}-t^{2}}}{\alpha^{2}-t^{2}}
\end{gathered}
$$

Thus, these coupled Fredholm integral equations reduced into decoupled Fredholm integral equations of second kind.

Define a constant function $\delta$, which depends upon the elastic properties of the medium. $\delta=K_{14}$ and $\delta_{1}=K_{14} K_{15} \sum_{n-1}^{\infty}(-1)^{n-1}\left(K_{14}^{n}\right) r_{1}^{n-1}$.

Where $\delta=K_{14}=K_{8} / K_{7}$ and $K_{15}=1+K_{5} / K_{7}$. Then the numerical values of $\delta=0$, when orthotropic medium becomes same as the medium of $y \geq 0$ otherwise, $0 \leq \delta<1$. Thus, we assume the expansion for $\mathrm{h}(\mathrm{t})$ as

$$
h(t)=\sum_{n-0}^{\infty} \delta^{m} h_{m}(t)
$$

and Substitute in equation (33) and then equate the coefficients of $\delta^{n-1}$ on both sides of equation, get

$$
\begin{gathered}
h_{0}(t)=-P_{14}(t) \\
h_{1}(t)=-K_{15} I_{0}(t)
\end{gathered}
$$

$$
\begin{gathered}
h_{2}(t)=-K_{15}^{2} I_{1}(t)-r_{1} K_{15} I_{0}(t) \\
h_{3}(t)=-K_{15}^{3} I_{2}(t)-r_{1} K_{15}\left(1+K_{15}\right) I_{1}(t)-r_{1}^{2} K_{15} I_{0}(t)
\end{gathered}
$$

Where $I_{0}(t), I_{1}(t), I_{2}(t)$ are calculated by,

$$
\begin{gathered}
I_{n}(t)=\int_{0}^{b} K_{0}\left(x_{1}, t\right) I_{n-1}(x) d x, n>1 \\
I_{0}(t)=\int_{0}^{b} K_{0}\left(x_{1}, t\right) P_{14}(x) d x
\end{gathered}
$$

Now substituting for equation (31) into equation (32), then simplifying and rearranging and get

$$
\begin{aligned}
& g(t)=P_{15}(t)+\frac{K_{13} K_{15}}{\pi K_{12} \sqrt{b^{2}-t^{2}}}\left[\delta I_{0}(t)+\sigma^{2} I_{1}(t) K_{15}-\right. \\
& \left.r_{1} I_{0}(t)-\delta^{3} K_{15}^{2} I_{0}^{2}(t)-r_{1}\left(1+K_{15}\right) I_{1}(t)-r_{1} I_{1}(t)+\ldots\right]
\end{aligned}
$$

With

$$
P_{15}(t)=P_{13}(t)+\frac{K_{13} K_{15}}{\pi K_{12} \sqrt{b^{2}-t^{2}}} \int_{0}^{b} \frac{P_{13}(x) \sqrt{b^{2}-x^{2}}}{x^{2}-t^{2}} d x
$$

$$
I^{n}(t)=\int_{0}^{b} \frac{x \sqrt{b^{2}-x^{2}}}{x^{2}-t^{2}} I_{n}(x) d x, n=0,1,2, \ldots
$$

As both the mediums i.e. lower and upper half planes become similar isotropic medium then the problem reduces to simple crack opening in infinite medium under self-equilibrating systems of surface forces which will be equal and constant.

\subsection{Physical Quantities}

The questions of physical interest in fracture design criterion are stress and the crack opening displacement components. Physical quantities are evaluated through equations (3) and (4) for value of integral $x>b$. For the evaluation of the integral, firstly substitute the value of $\phi$ and $\psi$ from equation (26), (27) in equation (3) and find integral equation, i.e.

$$
\begin{aligned}
& \sigma_{x y}\left(x, 0^{+}\right)-\sigma_{x y}\left(x, 0^{-}\right)=-2 \frac{\left(1-r_{1}\right)}{\pi}\left[x K_{12} \int_{0}^{b} \frac{g(t) d t}{t^{2}-x^{2}}-\right. \\
& \left.x K_{12} \int_{0}^{\infty} \frac{P_{4}(t) d t}{t^{2}-x^{2}}+\left(1+r_{1}\right) K_{13} P_{5}(x)-\frac{P_{6}(x)}{\left(1+r_{1}\right)}\right], \quad b \leq x<\infty
\end{aligned}
$$

Similarly, substitute the value of $\phi$ and $\psi$ from equation (23), (24) in equation (4) and find integral equation, i.e.

$$
\begin{aligned}
& \sigma_{y y}\left(x, 0^{+}\right)-\sigma_{y y}\left(x, 0^{-}\right)=\frac{2}{\pi}\left[K_{11} \int_{0}^{b} \frac{h(t) d t}{t^{2}-x^{2}}-\frac{2 K_{11}}{\pi}\right. \\
& \left.\left(\int_{b}^{\infty} \frac{P_{5}(t) d t}{t^{2}-x^{2}}\right)+K_{10} P_{4}(x)-P_{7}(x)\right], b \leq x<\infty
\end{aligned}
$$




\subsection{Displacement Components}

To evaluate $u_{x}\left(x, 0^{ \pm}\right), u_{y}\left(x, 0^{ \pm}\right)$using continuity conditions equation (4), (5). Then we find the values of integral

$$
\begin{aligned}
& u_{x}\left(x, 0^{+}\right)-u_{x}\left(x, 0^{-}\right)=\int_{x}^{b} g(t) d t+P_{4}(b)-P_{4}(x), 0 \leq x<b \\
& u_{y}\left(x, 0^{+}\right)-u_{y}\left(x, 0^{-}\right)=\int_{x}^{b} h(t) d t+P_{5}(b)-P_{5}(x), 0 \leq x<b
\end{aligned}
$$

where $\mathrm{h}(\mathrm{t})$ and $\mathrm{g}(\mathrm{t})$ are the solutions of Fredholm integral equations.

To evaluate $u_{y}\left(x, 0^{+}\right)+u_{y}\left(x, 0^{-}\right)$and $u_{x}\left(x, 0^{+}\right)+u_{x}\left(x, 0^{-}\right)$, we get

(a) For isothermal case $(y>0)$

$$
\begin{aligned}
& u_{x}(x, 0)=u_{x}\left(x, 0^{+}\right)=\frac{2(1+\eta)}{\pi E} \int_{b}^{\infty} \frac{(\sin \xi x)}{\xi}\left[A \xi^{2}-2(1-\eta) \xi B\right] d \xi \\
& =\frac{2(1+\eta)}{\pi E} \int_{b}^{\infty} \sin \xi x[A \xi-2(1-\eta) B] d \xi \\
& u_{y}(x, 0)=u_{y}\left(x, 0^{+}\right)=\frac{2(1+\eta)}{\pi E} \int_{b}^{\infty} \cos \xi x[A \xi+(1-\eta) B] d \xi
\end{aligned}
$$

(b) For isotropic case $(y<0)$

$$
\begin{gathered}
u_{x}(x, 0)=u_{x}\left(x, 0^{-}\right)=\int_{b}^{\infty}(\sin \xi x)\left[\xi a_{11}\left(C r_{1}^{2}-r_{2} D\right)-a_{12} \xi C\right] d \xi \\
=\int_{b}^{\infty}(\sin \xi x)\left[\xi C\left(r_{1}^{2} a_{11}-a_{12}\right)-\xi D a_{11} r_{2}\right] d \xi \\
u_{y}(x, 0)=u_{y}\left(x, 0^{-}\right)=\int_{0}^{\infty} \cos \xi x\left[r_{1} \xi C\left(a_{11} r_{1}^{2} a_{12}-a_{66}\right)+\right. \\
\left.\xi D\left(a_{12}+a_{66}-r_{2}^{2} a_{11}\right)\right] d \xi
\end{gathered}
$$

Use of above isotropic cases, evaluate the following $u_{x}\left(x, 0^{+}\right)+u_{x}\left(x, 0^{-}\right)=\int_{b}^{\infty}(\sin \xi x)\left[\xi C K_{51}+\xi D K_{61}\right] d \xi$

$+K_{0} \int_{0}^{\infty} \frac{P_{1}(\xi) \sin \xi x d \xi}{\xi}$

$u_{y}\left(x, 0^{+}\right)+u_{y}\left(x, 0^{-}\right)=\int_{0}^{\infty} \cos (\xi x)\left[\xi C K_{71}+\xi D K_{81}\right] d \xi(40)$

Where,

$$
\begin{gathered}
K_{51}=K_{1}+K_{0}\left\{1+2(1-\eta)\left(1-r_{1}\right)\right\} \\
K_{61}=K_{1}+K_{0}\left\{1+2(1-\eta)\left(1-r_{1}\right)\right\} \\
K_{71}=K_{0}\left\{1-2(1-\eta)\left(1-r_{1}\right)\right\}+r_{1}\left(r_{1}^{2} a_{11}-a_{12}-a_{66}\right)
\end{gathered}
$$

$$
K_{81}=a_{11}+a_{66}-a_{11} r_{2}^{2}+K_{0}(1-2 \eta)
$$

Now we shall make use of $\xi C=\left(K_{8} \phi-K_{6} \psi\right) / K_{9}$ and $\xi D=\left(K_{7} \phi-K_{5} \psi\right) / K_{9}$. Further,

$u_{y}\left(x, 0^{+}\right)+u_{y}\left(x, 0^{-}\right)=\int_{0}^{\infty} \cos (\xi x)\left[\xi A K_{0}+K_{0}(1-2 \eta) B\right] d \xi+\varepsilon$
$\int_{0}^{\infty} \cos (\xi x)\left[r_{1} \xi C\left(r_{1}^{2} a_{11}-a_{12}-a_{66}\right)+\xi D\left(a_{12}-a_{66}-r_{2}^{2} a_{11}\right)\right] d \xi$

$=\int_{0}^{\infty} \cos (\xi x)\left[\xi C K_{0}+r_{1}\left(r_{1}^{2} a_{11}-a_{12}-a_{66}\right)-K_{0}\left(1-r_{1}\right)\right.$

$\left.(1-2 \eta)+\xi D\left\{a_{11}+a_{66}-a_{11} r_{2}^{2}+K_{0}(1-2 \eta)\right\}\right] d \xi$

$$
\begin{aligned}
& u_{y}\left(x, 0^{+}\right)+u_{y}\left(x, 0^{-}\right)=\int_{0}^{\infty} \sin (\xi x)\left[K_{51} \frac{K_{8} \phi-K_{6} \psi}{K_{9}}+\right. \\
& \left.K_{61} \frac{K_{7} \phi-K_{5} \psi}{K_{9}}\right] d \xi+K_{0} \int_{0}^{\infty} \frac{P_{1}(\xi) \sin (\xi)}{\xi} d \xi \\
& u_{x}\left(x, 0^{+}\right)+u_{x}\left(x, 0^{-}\right)=\int_{0}^{\infty} \sin (\xi x)\left[\phi K_{a}-\psi K_{b}\right] d \xi+ \\
& K_{0} \int_{0}^{\infty} \frac{P_{1}(\xi) \sin (\xi)}{\xi} d \xi
\end{aligned}
$$

With $K_{a}=\frac{\left(K_{51} K_{8}+K_{61} K_{7}\right)}{K_{9}}$ and $K_{b}=\frac{\left(K_{51} K_{6}+K_{61} K_{5}\right)}{K_{9}}$ $u_{y}\left(x, 0^{+}\right)+u_{y}\left(x, 0^{-}\right)=\int_{0}^{\infty} \cos (\xi x)\left[K_{71} \frac{K_{8} \phi-K_{6} \psi}{K_{9}}+\right.$ $\left.K_{81} \frac{K_{7} \phi-K_{5} \psi}{K_{9}}\right] d \xi+\int_{0}^{\infty} \frac{P_{1}(\xi) \sin (\xi)}{\xi} d \xi$ $=\int_{0}^{\infty} \cos (\xi x)\left[\phi\left\{\frac{K_{71} K_{8}+K_{7} K_{81}}{K_{9}}\right\}-\left\{\frac{K_{71} K_{6}+K_{5} K_{81}}{K_{9}}\right\}\right] d \xi$ $+\int_{0}^{\infty} \frac{P_{1}(\xi) \sin (\xi)}{\xi} d \xi$

$$
=\int_{0}^{\infty}\left[\phi K_{c}-\psi K_{d}\right] d \psi+\int_{0}^{\infty} \frac{P_{1}(\xi) \sin (\xi)}{\xi} d \xi
$$

Where,

$$
\begin{gathered}
K_{c}=\left(K_{71} K_{8}+K_{81} K_{7}\right) / K_{9} \\
K_{d}=\left(K_{71} K_{6}+K_{81} K_{5}\right) / K_{9}
\end{gathered}
$$


The values of integral are evaluated by using the relation (23) and (24) for $\phi(\xi)$ and $\psi(\xi)$ and then evaluating the integrals

$$
\begin{aligned}
& u_{x}\left(x, 0^{+}\right)+u_{x}\left(x, 0^{-}\right)=\int_{0}^{\infty}(\sin \xi x)\left[K_{a} \frac{2}{\pi \xi} \int_{0}^{\infty} g(t)(1-\cos \xi t) d t\right. \\
& -\frac{2}{\pi \xi} \int_{0}^{\infty} \frac{P_{4}(1-\cos \xi t) d t}{\xi}+K_{b} \frac{2}{\pi \xi} \int_{0}^{b} \sin \xi t 2 l t \\
& \left.-\frac{2}{\pi} \int_{0}^{b} \frac{P_{5}(t) \sin (\xi t)}{\xi}\right] d \xi \\
& =\frac{2}{\pi} K_{a} \int_{0}^{b} g(t) d t \int_{0}^{\infty} \frac{(\sin \xi x)(1-\cos \xi t)}{\xi} d \xi+\frac{2}{\pi} K_{b} \int_{0}^{b} h(t) d t \\
& \int_{0}^{\infty} \frac{(\sin \xi t)(\sin \xi x)}{\xi} d \xi-\frac{2}{\pi} K_{b} \int_{b}^{\infty} P_{5}(t) d t \int_{0}^{\infty} \frac{(\sin \xi t)(\sin \xi x)}{\xi} d \xi \\
& +K_{0} \int_{0}^{\infty} \frac{P_{1}(\xi) \sin (\xi)}{\xi} d \xi \\
& u_{x}\left(x, 0^{+}\right)+u_{x}\left(x, 0^{-}\right)=K_{a} \int_{x}^{b} g(t) d t+\frac{K_{b}}{\pi} \int_{0}^{b} h(t) \\
& \log \left|\frac{x+t}{x-t}\right| d t+K_{a} P_{4}(b)-\frac{K_{b}}{\pi} \int_{0}^{\infty} P_{5}(t) \log \left|\frac{x+t}{x-t}\right| d t \quad(41) \\
& +K_{0} \int_{0}^{\infty} \frac{P_{1}(\xi) \sin (\xi x)}{\xi} d \xi
\end{aligned}
$$

$$
\begin{aligned}
& u_{y}\left(x, 0^{+}\right)+u_{y}\left(x, 0^{-}\right)=\frac{2}{\pi} K_{c} \int_{0}^{b} g(t) d t \int_{0}^{\infty} \frac{(\cos \xi x)(\sin \xi t)}{\xi} \\
& d \xi-\frac{2}{\pi} K_{d} \int_{0}^{b} h(t) d t \int_{0}^{\infty} \frac{(\cos \xi x)(1-\cos \xi t)}{\xi} d \xi- \\
& \frac{2}{\pi} K_{c} \int_{0}^{\infty} P_{4}(t) \int_{0}^{\infty} \frac{(\cos \xi x)(\sin \xi t)}{\xi} d \xi-\frac{2}{\pi} K_{d} \int_{0}^{\infty} P_{5}(t) d t \\
& \int_{0}^{\infty} \frac{(\cos \xi x)(1-\cos \xi t)}{\xi} d \xi+K_{0} \int_{0}^{\infty} \frac{P_{1}(\xi) \cos (\xi x)}{\xi} d \xi \\
& u_{y}\left(x, 0^{+}\right)+u_{y}\left(x, 0^{-}\right)=\frac{K_{c}}{\pi} \int_{x}^{b} g(t) d t+\frac{K_{d}}{\pi} \int_{0}^{b} h(t) \\
& \log \left|x^{2}-t^{2}\right| d t+K_{c} P_{4}(b)-\frac{K_{b}}{\pi} \int_{0}^{b} P_{5}(t) \log \left|x^{2}-t^{2}\right| d t ?(42) \\
& +K_{0} \int_{0}^{\infty} \frac{P(\xi) \cos (\xi x)}{\xi} d \xi
\end{aligned}
$$

\subsection{Special Case}

Consider the crack is opening due to constant and uniform surface forces shown as figure 3 and i.e. $p_{1}(x)=p_{2}(x)=p_{0}$. After use of constant, $P_{1}(\xi)=0$.

$$
Y-\text { axis }
$$

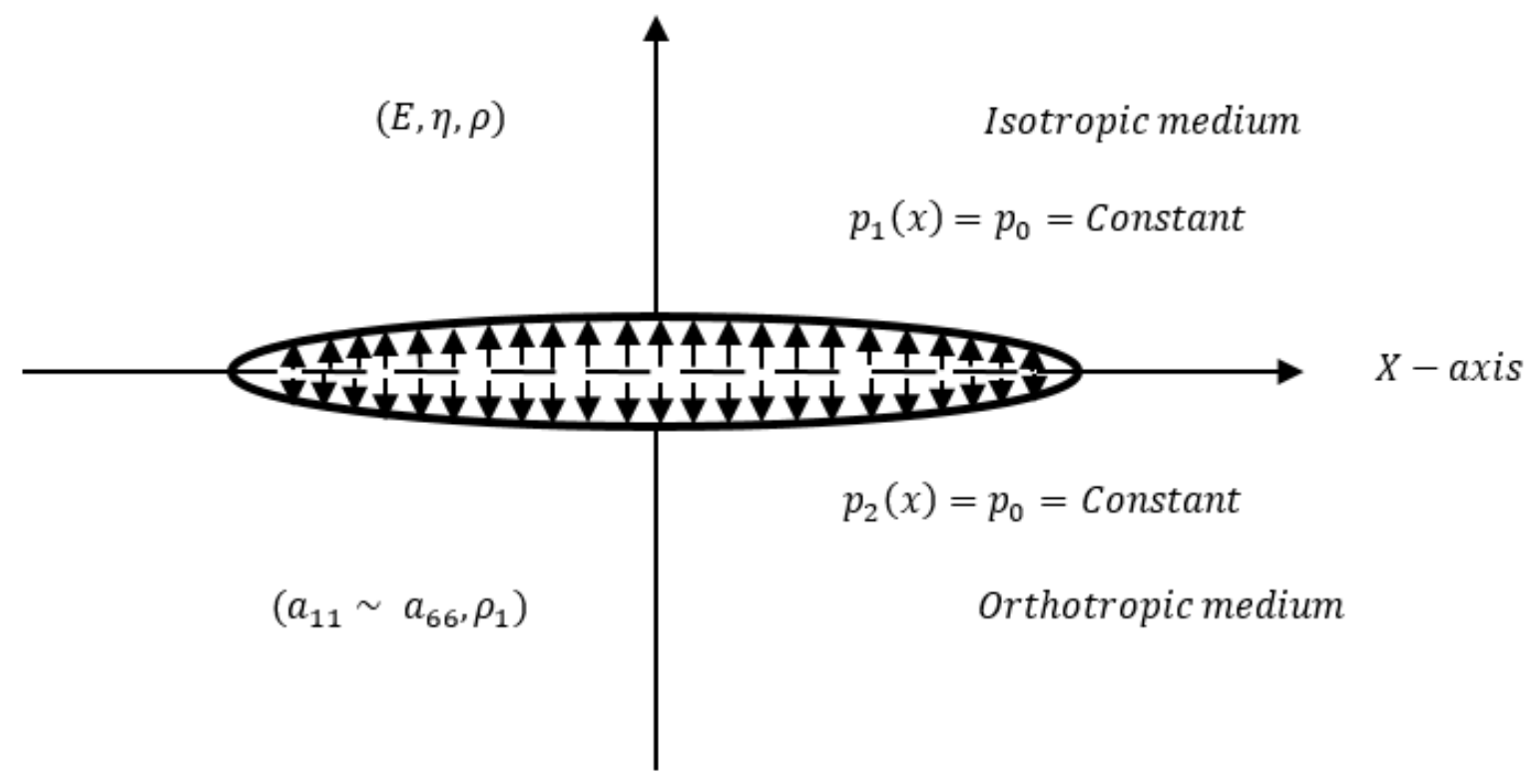

Figure 3. Geometry problem with Special Loading 
Through the value of $P_{1}(\xi)$, calculate the values, $P_{4}(x)=P_{5}(x)=P_{6}(x)=0, \quad P_{7}(x)=p_{0}, P_{8}(x)=p_{0} \quad$ and $P_{9}(x)=0$.

Now use of equation (12) and calculate the value of $P_{10}(x), P_{11}(x), P_{12}(t), P_{13}(t), P_{14}(t), P_{15}(t)$. i.e.

$$
\begin{gathered}
P_{10}(x)=p_{0}+K_{10} g(x) \\
P_{11}(x)=-K_{13} h(x) \\
P_{12}(t)=-\frac{t p_{0}}{\pi K_{11} \sqrt{b^{2}-t^{2}}} \\
P_{13}(t)=0 \\
P_{14}(t)=P_{12}(t) \\
P_{15}(t)=0
\end{gathered}
$$

Finally, calculate the value of $I_{0}(t)$, i.e.

$$
\begin{aligned}
& I_{0}(t)=\frac{t p_{0}}{\pi K_{11} \sqrt{b^{2}-t^{2}}} \frac{1}{\sqrt{b^{2}-t^{2}}} \log \left(\frac{b-t}{b+t}\right) \\
& {\left[\int_{0}^{b}\left(\frac{b+x}{b-x}\right)\left(\frac{\sqrt{b^{2}-x^{2}}}{x^{2}-t^{2}}\right)\right]}
\end{aligned}
$$

Be Thus we can easily evaluate $\mathrm{h}(\mathrm{t})$ and $\mathrm{g}(\mathrm{t})$ through equations (34), (35), (36), and (43). Knowing these two we can calculate the stress components and displacement component, in exact form.

\section{Conclusions}

From the above equations (7) and (8), the formulation of the problem "A Griffith Crack at the Interface of an Isotropic and Orthotropic Half Space Bonded Together" has been reduced to the dual integral equations (12), (13). In next, further equation (12), (13) have been solved and then further simplification yields to the Fredholm integral equations of second kind. Finally, we have solution of these Fredholm integral equations and then evaluate the physical quantities in terms of solution of Fredholm integral equations. Thus equation (29) and (30) are the coupled Fredholm integral equations and can easily be reduced to decoupled Fredholm integral equations of second kind. At last, we have taken a special case of uniform constant pressure and then equation (41), (42), and (43) are the exact form of the Stress components and Displacement Components of a Griffith crack at the interface of an Isotropic and Orthotropic half-space bonded together.

\section{REFERENCES}

[1] Williams M.L, "On the Stress Distribution at the Base of a Stationary Crack," J. Appl. Mech., vol. 24, no. 1, pp. 109-114, 1957. DOI: https://doi.org/10.1115/1.4011454

[2] Sneddon I. N., D. S. Berry, "The classical theory of elasticity," Encyclopedia of physics, vol. 9, pp. 1-126, 1958. DOI: 10.1007/978-3-662-43081-1_1

[3] Williams M. L., "The stresses around a fault or crack in dissimilar media," Bulletin of the seismological society of America, vol. 49, no. 2, pp. 199-204, 1959. DOI: https:// doi.org/10.1785/BSSA0490020199

[4] Erdogan F., "Stress distribution in bounded dissimilar materials with cracks," J. Appl. Mech., vol. 32, no. 2, pp. 403-410, 1965. DOI: https://doi.org/10.1115/1.3625814

[5] Rice J. R., Sih G. C., "Plane problems of cracks in dissimilar media," J. Appl. Mech., vol. 32, pp. 418-423, 1965.

[6] Muskhelishvili N. I., "Some basic problems in the mathematical theory of Elasticity," Noordhoff, 1965.

[7] Sneddon I. N., J. Tweed, "The stress intensity factor for a Griffith-crack in an elastic body in which body forces are acting,” Int. J. Fracture Mech., vol. 3, pp. 317-330, 1967. DOI: https://doi.org/10.1007/BF00182896

[8] Srivastava K. N., M. Lowengrub, "Finite Hilbert transform technique for triple integral equations with trigonometric Kernels," Proc. Roy. Soc. Edinb., vol. 68, no. 4, pp. 309-321, 1968. DOI: https://doi.org/10.1017/S0080454100 008463

[9] Sneddon I. N., M. Lowengrub, "Crack Problems in the Classical Theory of Elasticity," John Wiley \& Sons, 1st ed, 1969, pp. 1-221.

[10] Tweed J., "The distribution of stress in the vicinity of a Griffith crack in an elastic solid in which body forces are acting,” Int. J. Engng. Sci., vol. 7, no. 8, pp. 815-842, 1969. DOI: https://doi.org/10.1016/0020-7225(69)90063-9

[11] Lowengrub M., I. N. Sneddon, "The stress field near a Griffith crack at the interface of two bounded dissimilar elastic half planes," Int. J. Eng. Sci., vol. 11, no. 9, pp. 1025-1034, 1973. DOI: https://doi.org/10.1016/0020-7225 (73) $90015-3$

[12] Lal M., D. Pandey, "The axisymmetric thermo elastic stress distrubution in an infinite solid containing a crack," Proc. Nat. Acad. Sci., India, vol. 45(A), pp.175-180, 1975.

[13] Dwivedi A. P., Sharma J. P., "Pair of coplanar Barenblatt cracks at the interface of two bounded dissimilar elastic half palnes," Ganita. vol. 31, pp. 54-67, 1980.

[14] Kushwaha P. S., Chandra U., "The stress intensity factors for Griffith-crack(s) opened by thermal stresses in an infinite solid," Proc. 25th. Congress of ISTAM, pp. 130-138, 1980.

[15] Dwivedi A. P., J. P. Sharma, "N- Barenblatt cracks at the interface of two bonded dissimilar elastic half planes," Acta Ciencia Indica, vol. 7, pp. 25-33, 1981.

[16] Dwivedi A. P., Kushwaha S. P., B. I. Tripathi, "The stress field due to a system of Griffith cracks at the interface of 
two bonded dissimilar wedge," J.M.A.C.T., vol. 14, pp. 35-51, 1981.

[17] Erdogan F., Kaya A. C., P. F. Joseph, "The crack problem in bonded non-homogeneous materials," Trans. ASME, J. Appl. Mech., vol. 58, no. 2, pp. 410-418, 1991. DOI: https://doi.org/10.1115/1.2897201

[18] Willis R. J., "Fracture mechanics of interfacial cracks," J. Med. Phys. Solids, vol. 19, pp. 358-368, 1991.

[19] Tweed J., "The determination of the stress intensity factor of a partially closed Griffith crack," Int. J. Engng. Sci., vol. 8, no. 9, pp. 793-803, 1970. DOI: https://doi.org/10.1016/0
020-7225(70)90005-4

[20] Dwivedi A. P., R. K. Tripathi, "Two Griffith cracks opened by heated wedge in an infinite isotropic medium," Acta Ciencia Indica, 2001.

[21] Mahmoud M. El-Borai, Khairia El-Said El-Nadi, "The Parabolic Transform and Some Singular Integral Evolution Equations," Mathematics and Statistics, Vol. 8, No. 4, pp. 410 - 415, 2020. DOI: 10.13189/ms.2020.080406.

[22] Leontiev V. L., "Orthogonal Splines in Approximation of Functions," Mathematics and Statistics, vol. 8, no. 2, pp. 167 - 172, 2020. DOI: 10.13189/ms.2020.080212 